\title{
Search through a sequentially presented visual display
}

\author{
JAMES E. HOFFMAN \\ University of Delaware, Newark, Delaware 19711
}

\begin{abstract}
Several paradoxes in the visual information processing literature are reviewed. Two experiments utilizing a sequential presentation technique suggested a two-stage model of visual search in which a parallel initial stage guides the operation of a slower serial discrimination stage. A computer simulation provided a good quantitative fit to the results of both experiments. The two-stage model shows how the paradoxes may be resolved as well as providing a framework for integrating results from visual search and spatial selective attention research.
\end{abstract}

In normal environments, the human observer is usually faced with the necessity of recognizing several simultaneously available forms. Can these separate form inputs be recognized in parallel and independently, or is there a limitation in the visual information processing system? This question appears to be straightforward, but attempts to provide an answer have produced a literature that presents several fascinating paradoxes.

The first paradox revolves around the speed at which the subjects can search an array of characters for a particular target. Sperling, Budiansky, Spivak, and Johnson (1971) estimated that subjects can search a display at the rate of 1 character $/ 8-12 \mathrm{msec}$. This high rate is obtained despite the presence of two slow stages: memory comparison and spatial selective attention. Sternberg (1966) proposed that a display character is compared to the target set via a serial, exhaustive, comparison process with a rate of approximately one comparison/ $40 \mathrm{msec}$. When several display elements are present, the subject may attend to them one at a time. This spatial attention mechanism has been studied by requiring subjects to report a cued item in a multielement display. Colegate, Hoffman, and Eriksen (1973) and Eriksen and Collins (1969) found that performance (accuracy and latency) continued to improve as the cue preceded the display by intervals as long as $\mathbf{2 0 0} \mathrm{msec}$. Thus, if memory comparison and selective attention are components of the search process, a primary question is why the slow operating

This research was supported by NIMH Grant 27110 . The author wishes to thank Fred Masterson, Carolyn Johnson, and Marilyn Shaw for helpful comments on earlier drafts of this paper. He also gratefully acknowledges the help in data analysis provided by Jack Gillis. Portions of the paper were presented at the meeting of the Eastern Psychological Association, Boston, April 1977. Requests for reprints should be addressed to the author at the Department of Psychology, University of Delaware, Newark, Delaware 19711. times of these two processes are not the limiting factor in determining search rate.

The second paradox concerns the apparently contradictory findings of different visual search experiments. Nickerson (1966) and Sternberg (Note 1) found that the latency of finding a memory set character in a display was well described by a model assuming a serial exhaustive comparison in short-term memory and a serial self-terminating search of the display. Other experiments have shown search latency to be independent of the number of characters in memory or in the display (Schneider \& Shiffrin, 1977; Shiffrin \& Schneider, 1977). Shiffrin and Schneider (1977) proposed that the critical variable is the consistency of mapping of particular forms to the target and background set. Under consistent mapping (CM) conditions, targets may be "automatically detected" so that latency will not depend on load. When target and background sets switch roles (varied mapping or VM), the subject will utilize a slow serial comparison of memory set items to display items.

In this paper, a model is proposed that accounts for both paradoxes as well as new ones raised by the experiments to be reported. This model proposes that search is a two-stage process in which a slow serial comparison stage is guided by a quick parallel evaluation of the entire display. This view is, of course, similar to Neisser's (1967) proposal of preattentive processes guiding the operation of a focal attention stage.

The departure point for the present experiments was a study by Eriksen and Spencer (1969) in which the elements of a visual display (letters) were presented sequentially at various rates. Accuracy in detecting a single target letter was independent of presentation rate but declined as the number of display letters increased. The finding that presentation rate was ineffective suggested that each display element is classified as a target or nontarget in parallel and independently (the independent channels 
model, or IC model). The decrease in accuracy with increases in the number of display items is attributable to increases in the probability that on trials when a target is absent, a "noise" item will generate a false alarm.

The IC model was further supported by Shiffrin and Gardner (1972), who showed that search accuracy was independent of presentation rate even when the spatiotemporal order of inputs was known in advance by the observer. Kinchla (1974) provided an elegant formalization of the IC model that gave an excellent account of Eriksen and Spencer's (1969) data as well as his own data on redundant targets. Further development was provided by Estes (1972), Gardner (1973), and Shiffrin and Geisler (1973). The success of this approach leaves little doubt that in some circumstances the subject's search behavior results from a parallel independent classification of the separate display items.

Search latency in the sequential presentation paradigm is also compatible with a parallel analysis of separate display forms. Sternberg and Scarborough (1969) presented a sequence of letters at the rate of $1 / 75 \mathrm{msec}$, and required an observer to detect the presence of a letter chosen from a memory set of one to five characters. Latency was a linear function of memory set size, with a slope of about $40 \mathrm{msec}$. They suggested that the target was detected by a serial exhaustive comparison to the memory set. This result fits the first paradox because the comparison process would require $200 \mathrm{msec}$ with a five-letter set and letters are arriving every $75 \mathrm{msec!}$ The target's latency did not vary with its temporal position in the sequence arguing against a queuing model. The relatively high accuracy ensured that items were not simply being skipped while the comparison stage was occupied. Sternberg and Scarborough concluded that several display elements were being compared simultaneously.

The present series of experiments had two goals. The first experiment was designed to test the generality of the IC model by examining search accuracy in the sequential presentation paradigm under varied mapping conditions. Shiffrin and Schneider (1977) and Sperling et al. (1971) found increases in accuracy with decreases in presentation rate. In both studies, decreasing rate was confounded with decreases in visual masking and increases in exposure duration. Thus, contrary to Eriksen and Spencer (1969), both studies found an effect of rate even in CM conditions. These results probably do not reflect limitations in processing capacity but, rather, limitations in the quality of the display representation (Norman \& Bobrow, 1975).

The second point of interest was the effect of presentation rate on detection latency. In particular, it was of interest to explore Sternberg and
Scarborough's (1969) suggestion that multiple display elements may occupy the comparison stage together. Varying presentation rate should reveal whether such a process holds for rates faster than 1 element $/ 75 \mathrm{msec}$. The second experiment examined detection latency in the sequential presentation paradigm under high-accuracy conditions.

The paradigm utilized here is shown in Figure 1. The display letters appear on the circumference of an imaginary circle, allowing variation in presentation rate without variation in exposure duration or visual masking. The possibility of lateral masking in these conditions was examined by Hoffman (Note 2), who showed that the spacing used here did not produce lateral masking. Since the target and background sets periodically switched roles, an effect of presentation rate was anticipated. Such an effect would reflect a resource limitation and argue against the IC model as a general description of search behavior.

\section{EXPERIMENT 1}

\section{Method}

Subjects. The author and three graduate students served as subjects.

Apparatus and Stimuli. Letters were $.23^{\circ}$ high and constructed by illuminating the appropriate points in a 6 by 5 matrix on a Tektronix 604 display monitor equipped with P-15 phosphor under control of a PDP-12 computer. Each point had a luminous directional intensity of 2.8. $\times 10-7$ cd on a dark background. Letters appeared equally spaced on the circumference of an imaginary circle $2.1^{\circ}$ in diameter centered on the fixation point.

Procedure. Each subject served in two practice and three experimental sessions. Each session consisted of three blocks of 320 trials. Each block was devoted to a memory set of one, two, or four letters. Within each block, the combination of five SOA values $(0,25,50,75$, and $100 \mathrm{msec})$ and positive or negative trial type occurred equally often in a random order. On positive trials, each member of the memory set occurred equally often in one of four temporal position pairs: $(1,2),(3,4),(5,6)$, and $(7,8)$. The particular member of the temporal position pair was chosen randomly.

The composition of the memory set changed within blocks as follows. The letters comprised three sets: (PVDH), (RBNF), (GXLM). Let $\mathrm{N}$ be the size of the memory set. The first $\mathrm{N}$ members of Set 1 served as the memory set for $N \times 40$ trials. Noise elements were chosen from Sets 2 and 3. Following this, the next $\mathbf{N}$ members of Set 1 became the memory set until Set 1 was exhausted, at which time Set 2 became the memory set with noise elements chosen from Sets 1 and 3 . Set 3 was not used for memory set elements. The advantages of this procedure are that the subject sees essentially identical displays independent of memory set size, and the members of the sets are well learned.

On each trial, the subject fixated a light point and initiated a trial with a microswitch. Eight letters appeared sequentially around the circumference of an imaginary circle (see Figure 1). The spatial order of letters was random, as was the spatial position of the target on positive trials. Positive latencies were measured from onset of the target element, while negative latencies were measured from onset of the last item in the sequence. Displays were not refreshed.

The subject pressed one of two microswitches to indicate whether or not he detected a match between any display element 


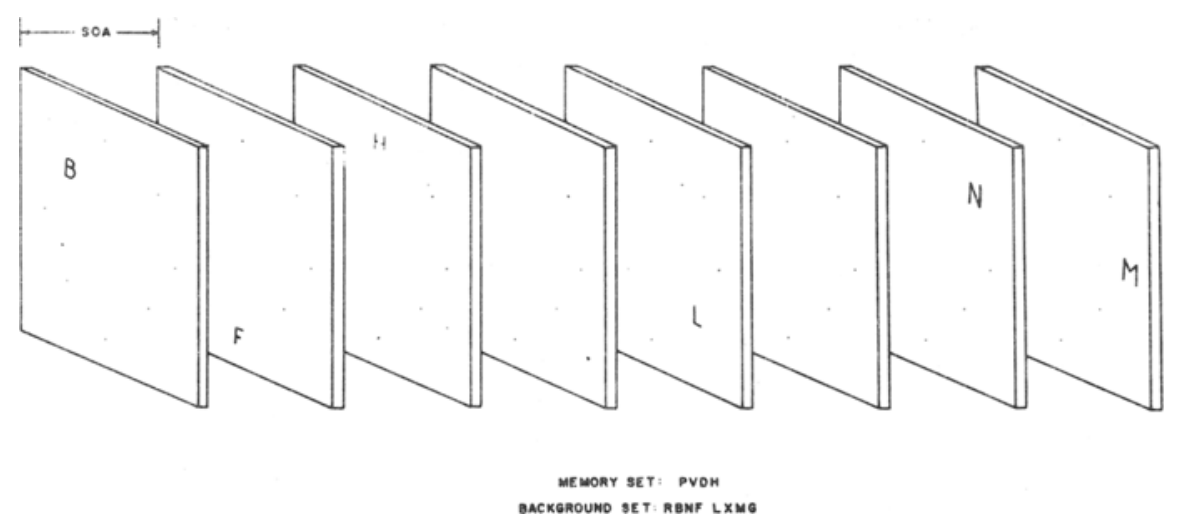

Figure 1. The sequential search paradigm. Each display letter appears in a different time frame.

and memory set item, and received immediate feedback on correctness of response and latency.

\section{Results and Discussion}

Figure 2 shows hit rate and false alarm rate as a function of SOA and memory set size. These results offer a clear answer to the conjecture that rate would be an effective variable when the target and background elements periodically switch roles. It appears that the probability of correctly detecting the target increases as presentation rate is decreased. In addition, larger memory set sizes lead to decreased detection accuracy, this affect appearing largely additive with rate. The false-alarm rate is generally low and, beyond $25 \mathrm{msec}$ SOA, shows little effect of presentation rate or memory set size.

Hit rate and false-alarm rate were used to compute $\mathrm{d}^{\prime}$ values assuming equal variance and normal distributions of signal and noise. A repeated measures analysis of variance revealed significant effects of rate $[F(4,12)=22.6, p<.001]$ and memory set size $[\mathrm{F}(2,6)=83.6, \mathrm{p}<.001]$. The interaction was not significant $[F(8,24)<1]$. We therefore conclude that the IC model is not a general description of search accuracy.

Correct latencies averaged over subjects are shown in Figure 3. Both positive and negative latencies increase with increasing memory set size. Positive latencies show little or no effect of rate, while negative latencies show a large decrease as presentation rate is decreased. The latter finding is really not surprising since, if the rate is slow enough, we are measuring the tiine to classify a single character (the last one in the sequence) vs. a set of eight characters in the 0-msec condition. By the same reasoning, it is surprising to find so little effect of rate on positive trials.

A separate analysis of variance was conducted on positive and negative latency data. For positive data, the only significant effect was memory set size $[\mathrm{F}(2,6)$
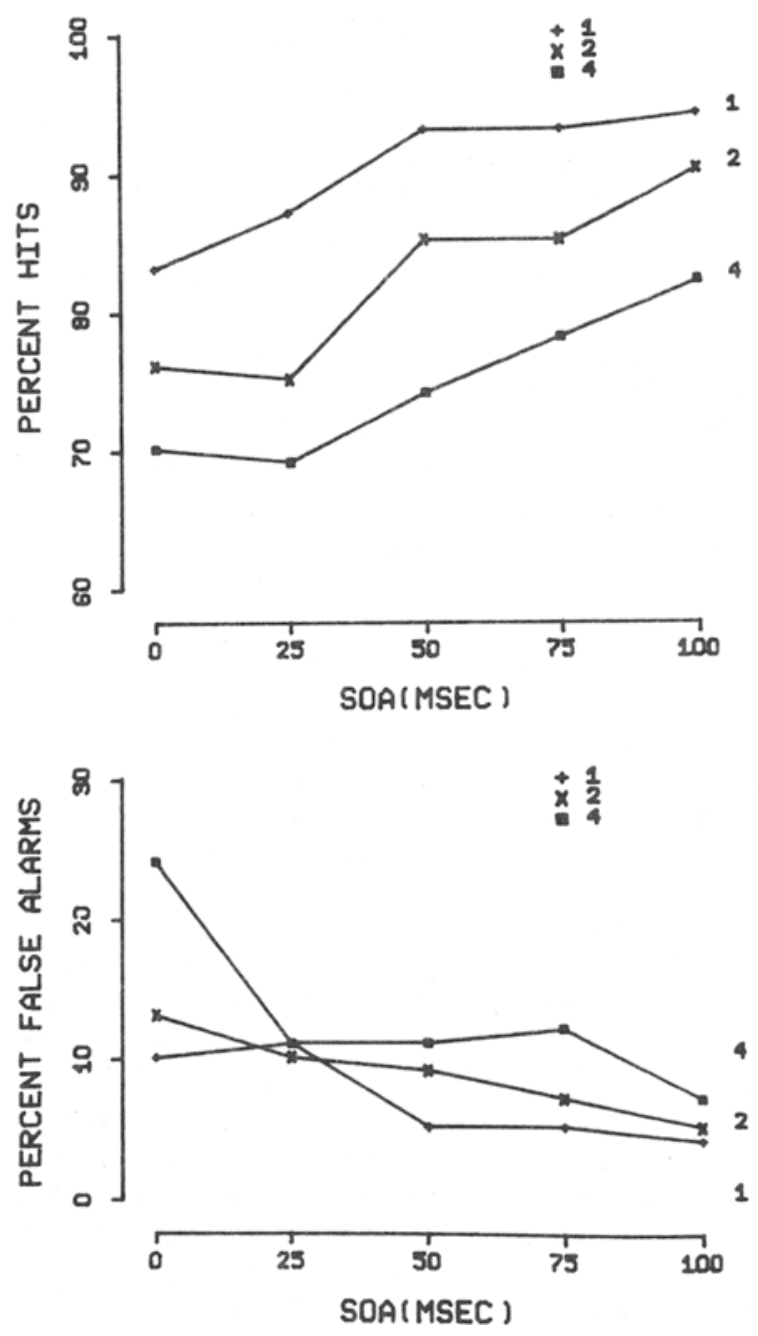

Figure 2. Hit rate and false-alarm rate as a function of the interval between characters. Parameter is the number of itenis in the memory set. Experiment 1. 

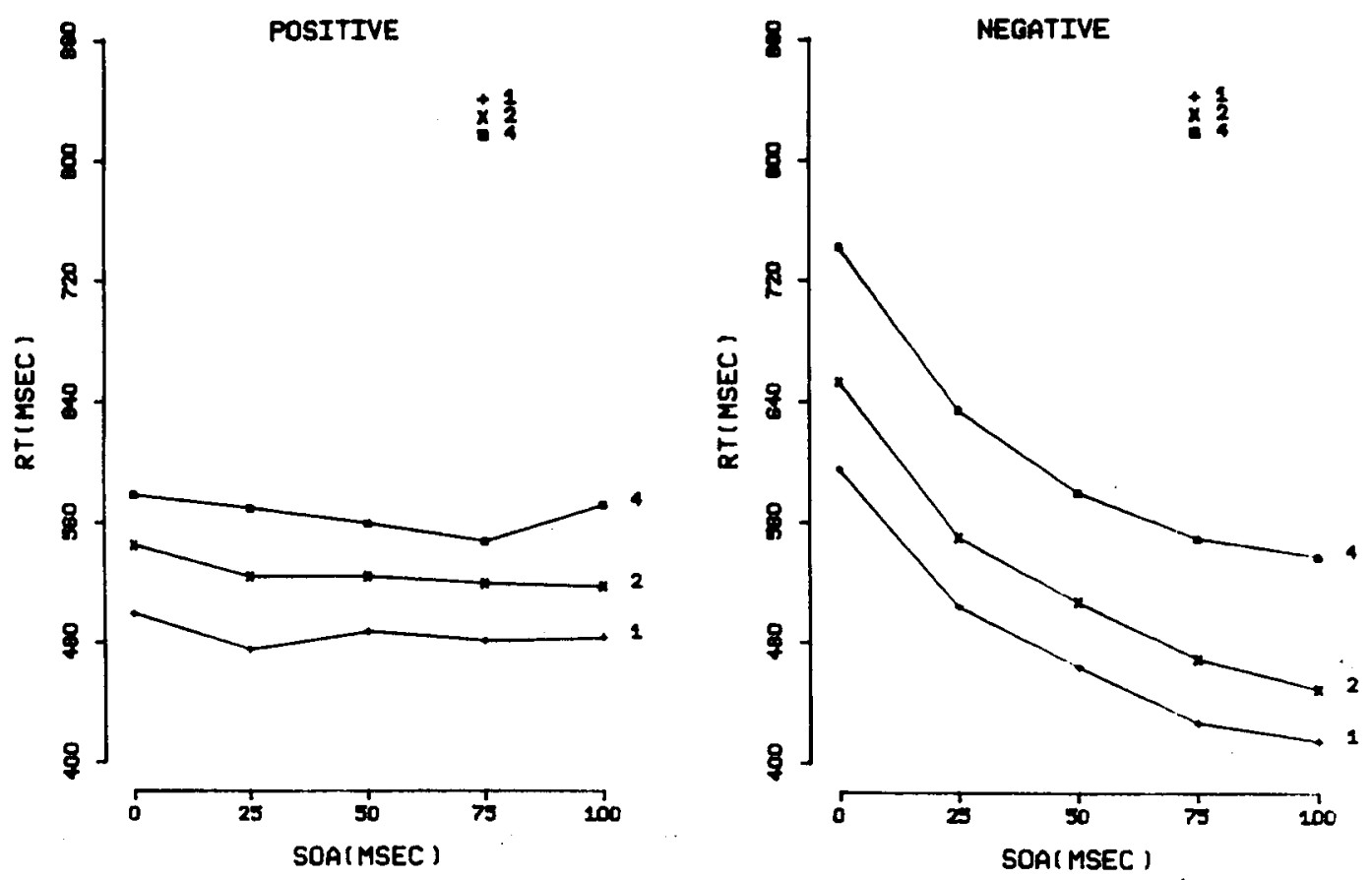

Figure 3. Average correct latency as a function of the interval between characters for positive and negative trials. Parameter is the number of items in the memory set. Experiment 1.

$=40.7, \mathrm{p}<.001]$. For negative trials, significance was obtained for memory set size $[\mathrm{F}(2,6)=19.2$, $\mathrm{p}<.003]$ and rate $[\mathrm{F}(4,12)=86.1, \mathrm{p}<.001]$. The interaction was not significant $(F<1)$.

One other aspect of the RT data bears mentioning. Notice that as the SOA value is increasing, the RT on negative trials is decreasing, apparently approaching asymptote at $100 \mathrm{msec}$. The asymptotic SOA may be interpreted as that time required to process a single character, since further increases in the time between subsequent characters does not lead to any reduction in RT. The data of Figure 3 indicate that the asymptotic SOA value on negative trials is independent of memory set size. By the reasoning outlined above, we could conclude that the time to process a display character is independent of memory set size.

A paradox is apparent, however, when one notes that RT is clearly dependent on memory set size. One way to resolve this paradox is to first assume a serial exhaustive comparison process producing a dependence of RT on memory set size. We would also have to assume that this comparison process is not the limiting factor on the rate at which characters can be processed. Sternberg and Scarborough (1969) came to the same conclusion by noting that search was much faster than would be expected on the basis of a slow memory-comparison process.

Sternberg and Scarborough suggested that one way a slow comparison process could keep up with a fast presentation rate would be to queue items arriving during a comparison. They showed, however, that there was no effect of the temporal position of the target in the sequence, contrary to a queuing theory. We examined our temporal position data in pairs $(1,2),(3,4),(5,6)$, and $(7,8)$ excluding the 0 SOA condition. Analysis of variance on mean RT revealed no significant effect of temporal position or its interaction with memory set size or rate (all Fs $<1$ ). Two of the subjects did, however, show a small increase in $\mathrm{RT}$ with increasing temporal position and in related experiments (Hoffman, Note 2) we have occasionally observed significant temporal position effects. These are probably due to subjects acting in accord with Bayes' theorem. At the slowest presentation rate, by the time the subject has examined the first seven positions and failed to find a target, the probability of a target's occurring is now $1 / 16$. This reduction in target probability with increasing temporal position is probably the reason that negative RTs are faster than positive at the large SOAs. We examine this possibility in the next experiment.

An analysis of hit rate as a function of temporal position revealed a clearer pattern of results. The main effect of temporal position was nearly significant $[F(3,9)=2.97, p<.09]$. The interaction of temporal position with memory set size $[F(6,18)=$ $3.8, \mathrm{p}<.01]$ and rate $[\mathrm{F}(9,27)=5.2, \mathrm{p}<.001]$ was significant. The interaction of memory set size and temporal position was due to the decrease in accuracy with increasing temporal position being larger for smaller memory set sizes. This result does not fit well 
within the queuing hypothesis discussed by Sternberg and Scarborough but is compatible with a Bayesian view of the subject. It does indicate that subjects may rapidly change their criteria within a time period of less than $1 \mathrm{sec}$.

In summary, these results show that the IC model may hold only for the situation in which the subject can search for the same character set consistently. This condition has been termed consistent mapping by Schneider and Shiffrin (1977). One may expect no effect of presentation rate in CM conditions when it is not confounded with visual masking effects (Eriksen \& Spencer, 1969). Under varied mapping conditions (VM), as in the present experiment, one may find large effects of rate primarily in terms of increasing hit rate with slower presentation rate.

The effects of presentation rate and memory set size on detection accuracy might be taken as evidence for a process which compares each memory set element to each display item seeking a match. Serialserial versions of this process (serial comparison through the display and memory) have been proposed by Nickerson (1966), Schiffrin and Schneider (1977) and Sternberg (Note 1). This type of model is not compatible with the finding of Experiment 1 that the asymptotic RT value is independent of memory set size. However, given the difficulty of interpreting RT when accuracy also varies in different conditions, Experiment 2 was designed

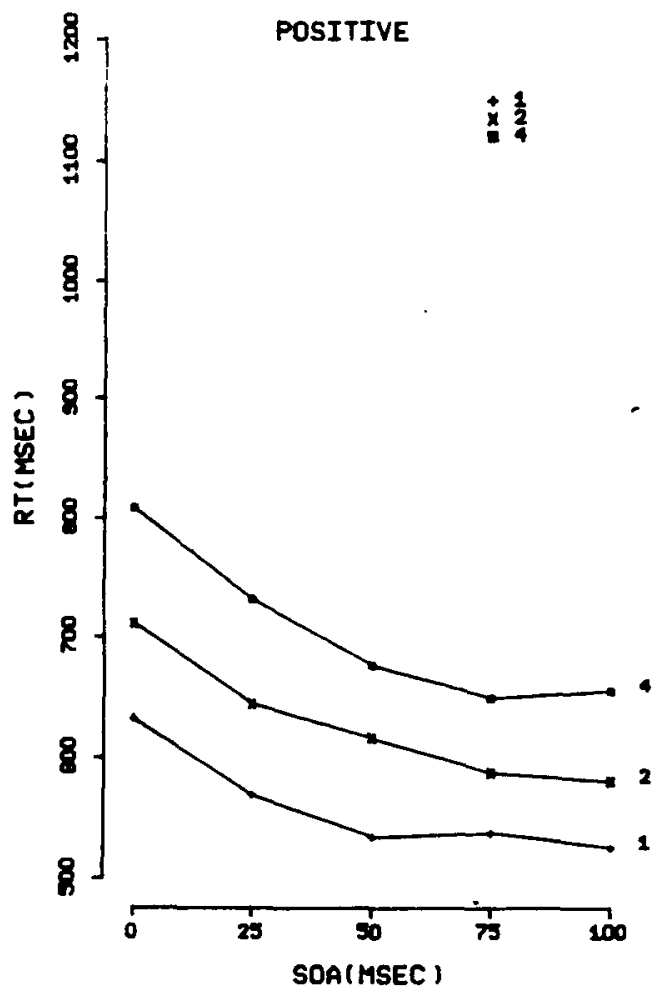

to measure RT in the sequential presentation paradigm when error rate is low.

In addition, an attempt was made to examine the subjects' sensitivity to the probability of targets' occurring in different temporal positions. Probability of target occurrence was high (.75) for the last two temporal positions which should lead to fast latencies for these positions, a finding opposite that of the last experiment as well as those reported by Hoffman (Note 2). Such a finding would indicate that the effects of temporal position can be attributed to variations in criterion rather than a queuing process.

\section{EXPERIMENT 2}

\section{Method}

All details of method were identical to those of Experiment 1, with these exceptions: Each character stayed on after it was presented so that the circle eventually "filled up" with characters. The entire display stayed on for $2 \mathrm{sec}$. The subjects were asked to be as accurate as possible. The second change was in the distribution of targets over temporal positions. Seventy-five percent of the targets occurred in Temporal Positions 7 and 8 , while the remaining targets were randomly distributed over the first six positions. Each subject served in three sessions.

\section{Results and Discussion}

Error rate was low; averaged over subjects, the largest error rate was $5 \%$ for a four-letter memory set

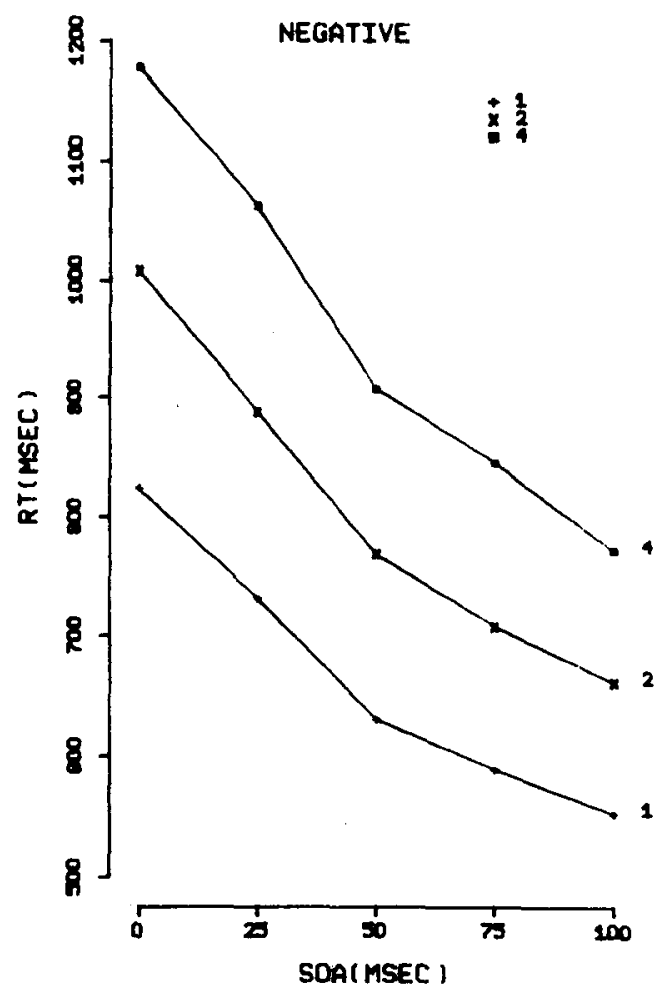

Figure 4. Average correct latency as a function of the interval between characters for positive and negative trials. Parameter is the number of items in the memory set. Experiment 2. 
at 0 msec SOA. Correct RT averaged over subjects is shown in Figure 4. Each point is based on approximately 384 observations. Most of the features of the RT data from Experiment 1 are replicated here. RT is approximately linear with respect to memory set size and the effect of SOA is much larger on negative than on positive trials. Notice that the SOA value at which positive RT functions approach asymptote is approximately the same, independent of memory set size. A clear asymptote is not apparent in the negative data.

Separate analyses of variance were conducted on the positive and negative latency data shown in Figure 4. For the positive latency data, significant effects were obtained for memory set size $[F(2,6)$ $=30.6, \mathrm{p}<.001]$, rate $[\mathrm{F}(4,12)=21.5, \mathrm{p}<.001]$, and their interaction $[F(8,24)=2.9, p<.02]$. The corresponding values for the negative data were 17.9 , 30.1 , and 5.8, all significant beyond .01.

The manipulation of probability of target occurrence in different temporal positions had the expected effect. Positive and negative latencies are similar at the slow rates, whereas in Experiment 1, negative RTs were faster than positive RTs at long SOAs. In addition, the temporal position function changed. Excluding the 0 SOA condition, the average RT for Temporal Positions 1-6 was $667 \mathrm{msec}$ and for Positions 7-8 was $597 \mathrm{msec}$. Contrary to findings in Experiment 1, the subjects were faster at later temporal positions. Again, this indicates that subjects are capable of dynamically changing their criteria for target detection in accord with target probability distribution. Shaw and Shaw (1977) found similar accuracy effects for probability of a target occurring in different spatial positions.

\section{GENERAL DISCUSSION}

The RT data of Experiment 2 are surprising in several respects. The most paradoxical finding is that although RT was clearly dependent on memory set size, the value at which the RT vs. rate function approached asymptote was not. That is, the memory comparison process presumed to underlie the dependence of RT on memory set size was not the limiting factor in search rate. Sternberg and Scarborough (1969) came to the same conclusion by noting that even with large memory sets, subjects could accurately locate targets in fast presentation sequences. A related finding is that of Shaw (1977), who had subjects search a string of letters in a prescribed order for a varying number of targets. She found that memory set size and string position of the target were additive in determining RT. If the subjects were serially searching the string in the prescribed fashion, their rate of search was independent of the size of the memory set. These findings are all incompatible with the serial-serial models described earlier.
The most straightforward modification of a strictly serial scanning model to accommodate these observations is the one proposed by Sternberg and Scarborough (1969); namely, allow more than one element at a time to be present in the comparison stage.

This model might be modified to account for the present data by assuming that there is a serial scanning device responsible for transferring items from iconic memory (the display representation) to the comparison process. An estimate of the transfer rate can be obtained as follows. Consider the positive RT function for a four-letter memory set shown in Figure 4. The total RT reduction going from 0 to $100 \mathrm{msec}$ SOA can be interpreted as the average search time required to locate the target in the 0 - $\mathrm{msec}$ condition. Assuming terminating search, the time/ item is approximately 44 msec. ${ }^{1}$ Applying the same reasoning to the negative data and assuming exhaustive search, we predict a total RT reduction going from 0 to $100 \mathrm{msec}$ SOA of $7 \times 44$, or $308 \mathrm{msec}$, which is somewhat smaller than the actual value of $400 \mathrm{msec}$. This underprediction holds for the other memory set sizes as well. The predictions of this model would be much worse for the data from Experiment 1. It also fails to account for the effects of memory set size on accuracy shown in Figure 2.

The principal difficulty that these data present for any serial model is the large difference between negative and positive latencies. Indeed, it is tempting to conclude that on positive trials the target is not found by a "blind" search but is, in fact, encountered early. This idea underlies the model to be described in the next section. Before presenting an alternative model, however, it might be useful to summarize the principal findings in the visual information processing literature. Any model with claims to generality must be capable of meeting this imposing set of constraints.

(1) Search performance decreases with increases in memory set size, display size, proximity of noise elements, rate of presentation, and signal/noise confusability. This last variable is particularly important, since low signal/noise confusability can eliminate the effects of the other variables (Estes, 1972).

(2) Consistent mapping of the same forms to the target set may eliminate the effects of the variables listed above (Shiffrin \& Schneider, 1977).

(3) The selection of display characters for detailed processing is under the subject's control and may require up to $200 \mathrm{msec}$ for completion (Colegate, Hoffman, \& Eriksen, 1973).

(4) The comparison of a single display item to memory is a serial exhaustive comparison (Sternberg, 1966), while the search through the display appears to be self-terminating (Sternberg, Note 1).

(5) The rate of search through a display is not 
limited by the duration of the memory comparison (this report; Shaw, 1977; Sternberg \& Scarborough, 1969).

The model presented in the next section meets the above set of constraints.

\section{A Visual Search Model}

Several years ago, Neisser (1967) outlined a twostage theory of visual search. An initial "preattentive" stage was responsible for segmentation of the scene and was parallel although error prone. It guided a second stage of focal attention which performed fine discriminations on a single object at a time. The model we present is closely related to Neisser's theory.

In outline, the model works as follows. Each item in the display is compared in parallel to all memory set items. The outcome is a similarity measure for each display item. Display characters are then serially transferred, in order of decreasing similarity, to a serial exhaustive comparison process. The transfer mechanism is assumed to be the same spatial selective attention mechanism studied in detail by Colegate, Hoffman, and Eriksen (1973) and Hoffman (1975). A detailed description follows.

Stage 1. Anderson (1973), Kinchla (1974), and Lappin (Note 3) have described parallel signal processing mechanisms based on statistical communication theory which accomplish signal detection by passing the signal through a filter. When one of several signals must be detected, the output of several filters are integrated which increases the noise in the detection process and lowers the signal/noise ratio. This idea forms the basis for an analogue to "preattentive processes" (Neisser, 1967). Assume that each memory set item can be represented as a filter and that the output of the filters are integrated. Increasing memory set size will lead to more noise in the output and a lower signal/noise ratio.

In particular let

$$
S_{i}=\sum_{j=1}^{M} x_{j}
$$

where $S_{i}$ is the similarity value for the $i^{\text {th }}$ display element and $M$ is the size of the memory set. $X_{j}$ is a normally distributed random variable with expected value and variance as follows:

$$
\begin{aligned}
E\left(X_{j}\right) & =0 \text { for a noise element } \\
& =\mu \text { for a target element }
\end{aligned}
$$

and

$$
\operatorname{VAR}\left(X_{j}\right)=\sigma^{2}
$$

Then, if the subject has $M$ letters in his memory set, the expected value and variance of the similarity value $S_{i}$ are as follows:

$$
\begin{aligned}
E\left(S_{i}\right) & =0 \text { for noise elements } \\
& =\mu \text { for a target element }
\end{aligned}
$$

and

$$
\operatorname{VAR}\left(S_{\mathrm{i}}\right)=\mathrm{M} \sigma^{2}
$$

Thus, the signal/noise ratio, analogous to the d' measure of signal detection theory, is

$$
\mathrm{S} / \text { No }=\mu / \sqrt{\mathrm{M} o^{2}} \text {. }
$$

In other words, the signal-to-noise ratio of the similarity value goes down with increasing memory set size.

The subject could, at this point, make a decision. The optimal procedure is to sum the similarity values corresponding to each display element and compare the sum to a criterion. Kinchla (1974) described this model and showed that it fit the Eriksen and Spencer (1969) data as well as his own data on redundant targets. Thus, a subject responding on the basis of Stage 1 analysis will behave as CM subjects do.

When the signal/ratio is low, as might happen in VM circumstances or when signal and noise items are similar, responding on the basis of Stage 1 would produce a high error rate. This error rate could be reduced by submitting items to a second, slower, more accurate discrimination process. We assumed that a candidate list was formed by comparing each similarity value to a criterion. All those items passing the criterion were placed on the candidate list. Items not placed on the candidate list received no further processing.

Transfer. Candidates are transferred to Stage 2 processing by a process of selective attention. That item currently having the highest similarity value is transferred first. Transfer time is a random variable having a rectangular distribution on the interval 0-200 msec. This assumption fits reasonably well with the data on leading indicators reviewed earlier. Once transfer is complete, transfer of another element may begin (assuming another candidate currently exists). Thus the transfer of or attention to display items can be conducted in parallel with memory comparison. However, if a display item completes transfer while a preceding one is still undergoing Stage 2 processing, the second one must queue. Due to this arrangement, the asymptotic SOA value will depend on the slower of two processes: the attentional mechanism or Stage 2 comparison. In the 
present experiments, the duration of the attention mechanism is longest leading to an asymptotic SOA value that is not dependent on memory set size.

Notice that if the signal/noise ratio is high the signal will, in general, be the first item transferred and there will be very little effect of rate on positive trials. On negative trials, however, the entire list must be examined, leading to large savings in RT with sequential presentation.

Stage 2. Stage 2 is a serial exhaustive comparison process with a comparison rate of one item $/ 30 \mathrm{msec}$ (this value was estimated from a separate single probe experiment). Only one item at a time can be present in this stage. At the end of an exhaustive memory comparison, if a match has been found, a positive response is initiated, otherwise a new display item is entered until either all comparisons have been made or limitations of iconic memory prevent any further comparisons. Stage 2 is assumed to be error free. Thus the main sources of errors (misses) are the failure of signal items to gain admission to the candidate list and the elimination of items from the candidate list when their waiting time exceeds iconic memory duration. False alarms, which were generally low in Experiment 1, are assumed to be guesses.

The simulation. These assumptions were incorporated into a computer program and fit to the data of Experiments 1 and 2. Free parameters were estimated by a nonlinear/least-squares technique. For Experiment 1, the free parameters were:

$\mu$ : The mean of the signal distribution for a single "filter" in Stage 1. The mean of the noise distribution was 0 .

B: The base RT which was the sum of all times not accounted for in the model such as motor components, etc.

IM: Iconic memory duration: The maximum time that items could reside on the candidate list before "fading." The criterion for admission to the candidate list was assumed to be 1.6 standard deviation units to the left of the mean of the signal distribution which would allow approximately $95 \%$ of the signals to be candidates for Stage 2 processing.

For each condition of Experiment 1, the computer simulated 160 trials, drawing from normal distributions with the appropriate mean and variance, sorting the candidate list, and eliminating items whose wait time was too long. The fit of the model to the RT data of Experiment 1 is shown in Figure 5. It is clear that a model with only three free parameters gives a good account of the 30 data points.
The standard error of the predicted values is 23.2 msec. The predicted hit rate with the same parameters is shown in Figure 6 along with the observed values. The model gives a good account of the effects of the independent variables on accuracy, except for a consistent overprediction for a oneelement memory set. The standard error of prediction is 6.1 . The estimated values were $\mu=1.9, \mathrm{~B}=$ $331 \mathrm{msec}$, and IM = $187 \mathrm{msec}$.

The identical model was fit to the data of Experiment 2, Figure 4, except that iconic memory duration was not a factor, reflecting the long exposure durations used. This two-parameter model produced the predicted values shown in Figure 7 . The fit is quite good, producing a standard error of $31.3 \mathrm{msec}$. The parameter values obtained here were $\mu=1.6$ and $\mathrm{B}=375$.

We conclude that a two-stage search model gives a good account of both accuracy and latency measures in a sequential search experiment. In addition, the same model predicts latencies obtained in a second experiment in which display duration is long and accuracy high. The assumptions of the model appear to satisfy the set of five constraints discussed earlier.

\section{CONCLUDING DISCUSSION}

In this paper, we have pointed out several paradoxes in the visual information processing literature.

(1) Visual search can proceed at a time/character rate that is faster than would be expected on the basis of a memory comparison process that can require some $200 \mathrm{msec}$ for a five-item set (Sternberg \& Scarborough, 1969) and a selective attention process that may also require some $200 \mathrm{msec}$ of operation time. Our results, as well as those of Shaw (1977), show that memory comparison time is not the limitation on search rate.

(2) Search behavior may or may not show effects of rate in different experiments. The critical variables determining the subject's behavior appear to be the practice he has had in looking for particular targets as well as the physical similarity of target and background elements.

These paradoxes can be resolved in terms of the two-stage search model proposed in this paper. The high speed of search relative to the operation time of several slow components is possible because of an initial parallel evaluation which can reject display elements quickly if they bear little similarity to the target set. With practice or with highly dissimilar target and background sets, the subject may base his detection decision on this parallel evaluation and will appear to be a parallel processor (Eriksen \& Spencer, 1969) or capable of "automatic detection" (Schneider \& Shiffrin, 1977; Shiffrin \& Schneider, 1977). 

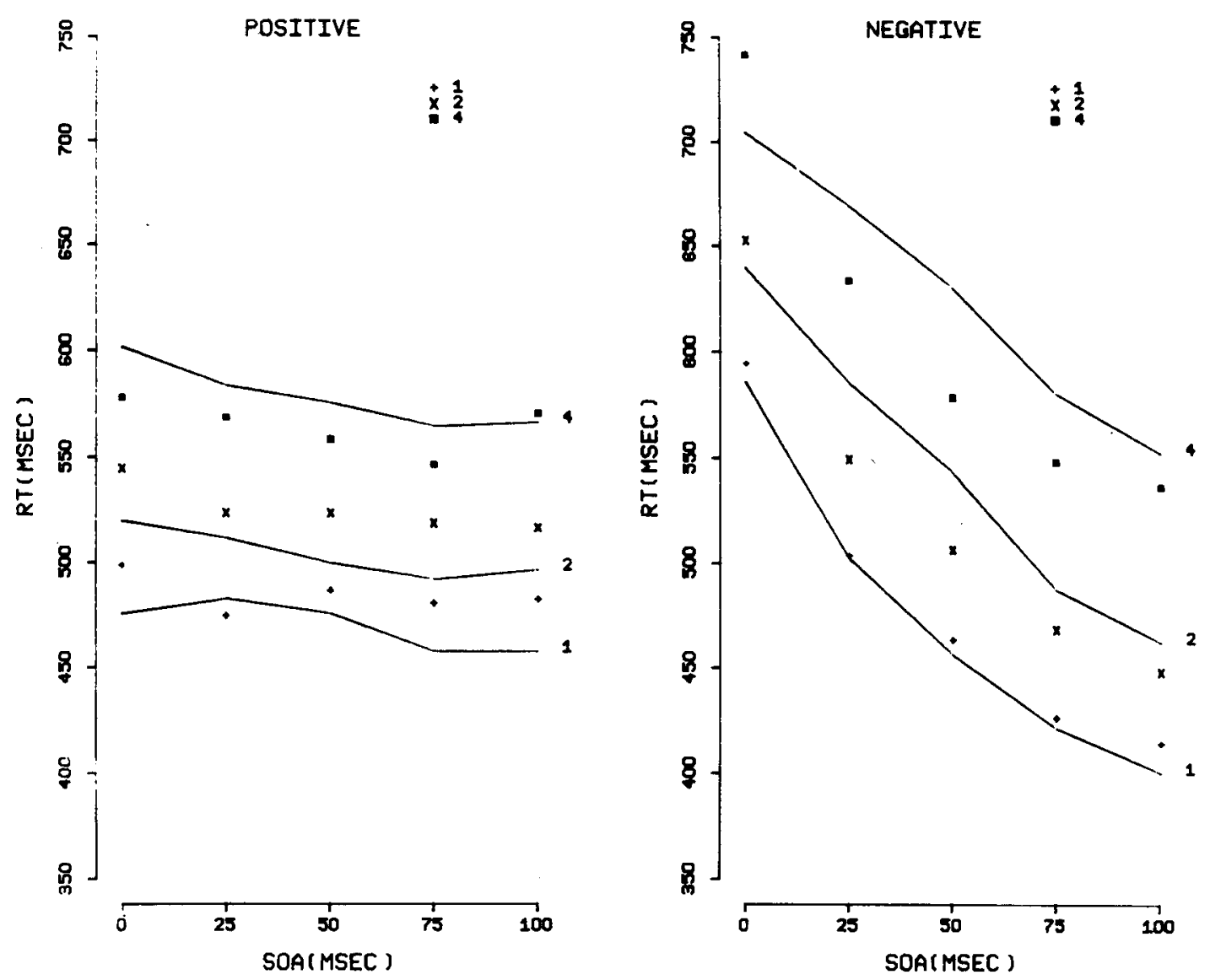

Figure 5. Predictions of the two-stage model (solid lines) and observed latencies (data points) for the data of Experiment 1.

When these conditions do not prevail, as in this experiment and other varied mapping procedures, the subject can reduce the error rate associated with a Stage 1 decision by employing a slower discrimination process. This Stage 2 analysis is directed at individual display characters and is guided by the Stage 1 analysis. In this case, the subject will obtain benefit from a sequential presentation, primarily because the directing of attention to individual display elements is a relatively slow process. Thus, the principal limitation on rate of information processing is not the duration of a serial comparison process is short-term memory, contrary to the serialserial model, but an attentional mechanism responsible for transfer of display items to the comparison process.

We tentatively identify Stage 1 processing with the identification of each display element. Given the highly spatially parallel nature of the visual system, which apparently provides for independent feature analysis across the entire retina, it is reasonable to assume that many separate forms make contact with corresponding long-term memory representation in parallel. Highly similar noise forms will also activate target representations to some degree. Thus the degree of activation of a set of long-term memory locations may serve as a "noisy" basis for a decision.

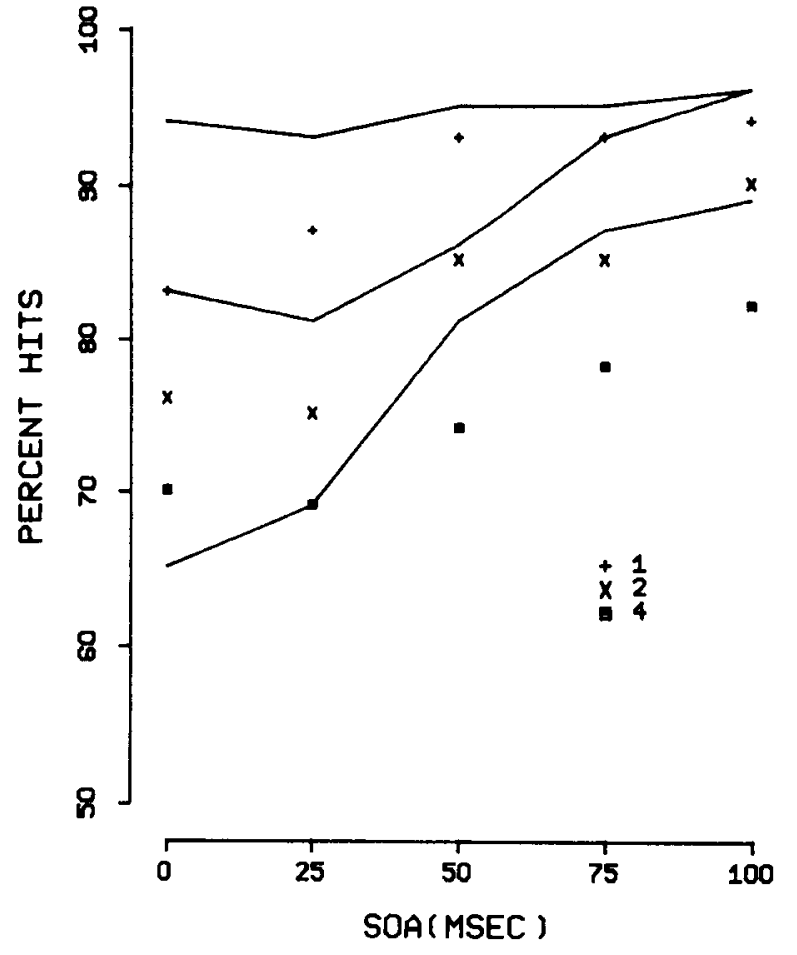

Figure 6. Predicted hit rate for the two-stage model (solid lines) using the parameters estimated from the fit to the latency data and observed hit rate (data points) for Experiment 1. 

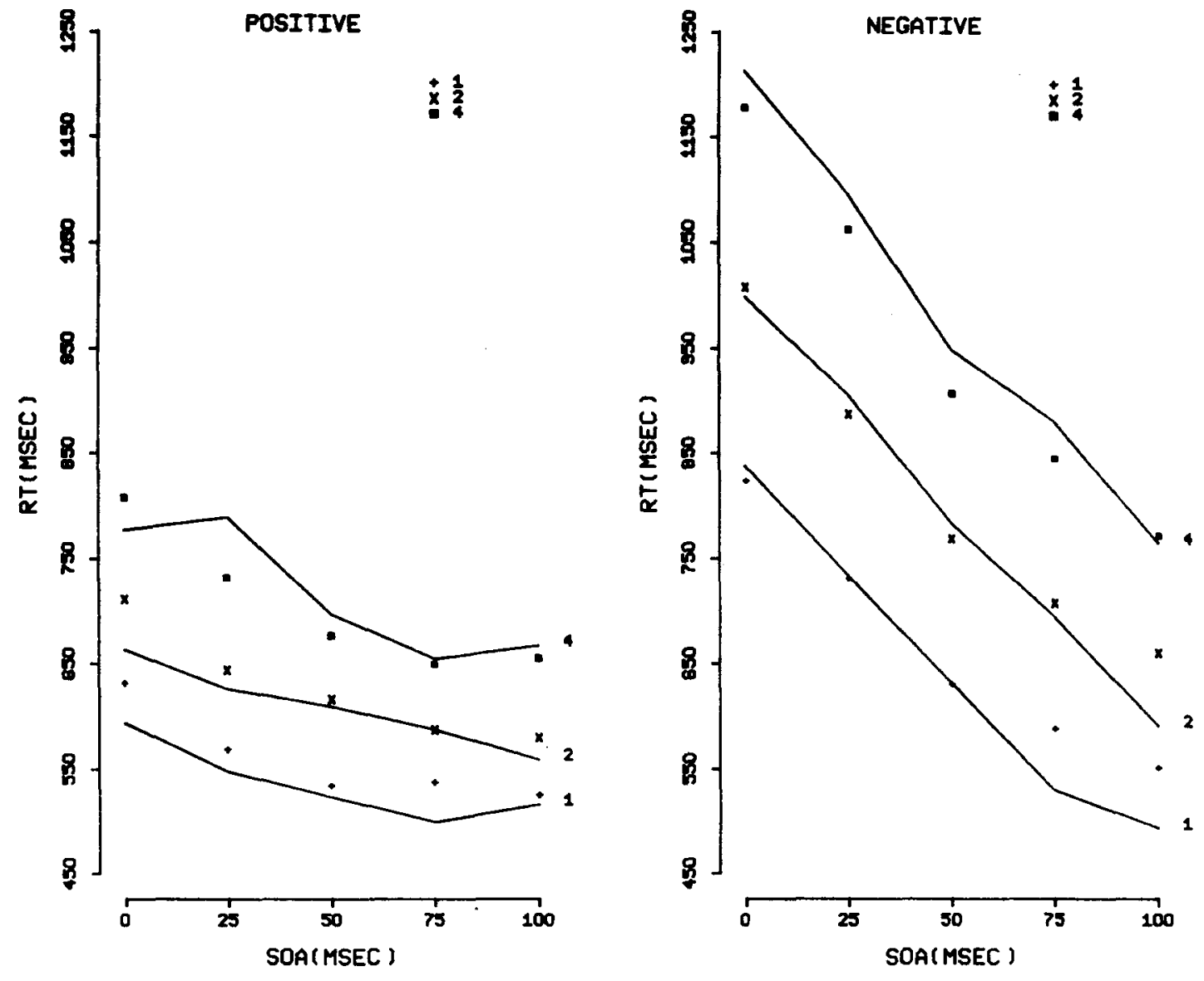

Figure 7. Predictions of the two-stage model (solid lines) and observed latencies (data points) for the data of Experiment 2.

Attention to a particular spatial position is equivalent to reducing the total noise in the decision process, allowing for a slower serial examination of activity in those memory locations corresponding to the target set. This recognition stage corresponds to the memory comparison process in Sternberg's (1969) taxonomy.

This is, of course, speculation, but it provides a framework which may prove useful in understanding several aspects of visual search: stimulus identification, spatial selection, and discrimination.

\section{REFERENCE NOTES}

1. Sternberg, S. Scanning a persisting visual image versus a memorized list. Paper presented at the meeting of the Eastern Psychological Association, Boston, April 1967.

2. Hoffman, J. E. A two-stage model of visual search. Paper submitted for publication.

3. Lappin, J. S. The relativity of choice behavior and the effect of prior knowledge on the speed and accuracy of recognition. Paper presented at the 1976 Conference on Mathematical and Theoretical Psychology, Bloomington, Indiana, April 1976.

\section{REFERENCES}

Anderson, J. A. A theory for the recognition of items from short memorized lists. Psychological Review, 1973, 80, 417-438.
Colegate, R. L., Hoffman, J. E., \& Eriksen, C. W. Selective encoding from multielement visual displays. Perception \& Psychophysics, 1973, 14, 217-224.

ERIKSEN, C. W., \& Collins, J. F. Temporal course of selective attention. Journal of Experimental Psychology, 1969, 80, 254-261.

ERIKsen, C. W., \& Spencer, T. Rate of information processing in visual perception: Some results and methodological considerations. Journal of Experimental Psychology, 1969, 79 (2, Pt. 2).

Estes, W. K. Interactions of signal and background variables in visual processing. Perception \& Psychophysics, 1972, 12, 278-286.

GARDNER, G. T. Evidence for independent parallel channels in tachistoscopic perception. Cognitive Psychology, 1973, 4, 130-155.

Hoffman, J. E. Hierarchical stages in the processing of visual information. Perception \& Psychophysics, 1975, 18, 348-354.

Kinchla, R. A. Detecting target elements in multi-element arrays: A confusability model. Perception \& Psychophysics, $1974,15,149-158$.

NeISSER, U. Cognitive psychology. New York: Appleton-CenturyCrofts, 1967.

NiCKERSON, R. S. Response times with a memory dependent decision task. Journal of Experimental Psychology, 1966, 72, 761-769.

Norman, D., \& Bobrow, D. On data-limited and resourcelimited processes. Cognitive Psychology, 1975, 7, 44-64.

Schnemer, W., \& Shiffrin, R. M. Controlled and automatic human information processing: I. Detection, search, and attention. Psychological Review, 1977, 84, 1-66.

ShAw, M. L. Reaction time in reading a tachistoscopic display 
for a memory set item. Perception \& Psychophysics, 1977. 21. 15-25.

Shaw. M. L.. \& Shaw. P. Optimal allocation of cognitive resources to spatial locations. Journal of Experimental Psychology: Human Perception and Performance, 1977, 3 , 201-211.

ShIfFRIN, R. M., \& Gardner, G. T. Visual processing capacity and attentional control. Journal of Experimental Psychology, $1972,93,72.82$

Shiffrin, R. M., \& Geisler. W. S. Visual recognition in a theory of information processing. In R. Solso (Ed.), The Loyola Symposium: Contemporary viewpoints in cognitive psychology. Washington, D.C: Winston, 1973.

Shiffrin, R. M., \& SCHNEIDER, W. Controlled and automatic human information processing: II. Perceptual learning, automatic attending and a general theory. Psychological Review, 1977, 84, 127-190.

Sperling, G., Budiansky, J., Spivak, J., \& Johnson, M. C. Extremely rapid visual search: The maximum rate of scanning letters for the presence of a numeral. Science, 1971, 174, 307-311.
STERNBERG, S. High-speed scanning in human memory. Science, 1966, 153, 652-654.

Sternberg, S. Memory scanning: Mental processes revealed by reaction time experiments. American Scientist, 1969, 57. 421.457.

Sternaerg, S., \& Scarborough, D. L. Parallel testing of stimuli in visual search. In Proceedings of the International Symposium on Visual Information Processing and Control of Motor Activity. Sofia: Bulgarian Academy of Sciences, 1969.

\section{NOTE}

1. The assumptions involved in this model are that (1) a $100-\mathrm{msec}$ SOA corresponds to presentation of a one-letter display, (2) the effect of display size on RT is linear, and (3) search is self-terminating on positive trials and exhaustive on negative trials.

(Received and accepted for publication November 1, 1977.) 